\title{
Hacia un modelo de medición de la ciencia desde el Sur Global: métricas responsables
}

Towards a model of metrics of science from global south: responsible metrics

\section{Gabriel Vélez Cuartas}

Universidad de Antioquia. Facultad de Ciencias Sociales y Humanas. Grupo de Redes y Actores Sociales, Colombia CoLav, Universidad de Antioquia - AmeliCA Métricas, Colombia gjaime.velez@udea.edu.co

\author{
Alejandro Uribe-Tirado \\ Universidad de Antioquia. Escuela Interamericana de Bibliotecología. Grupo Información, \\ Conocimiento y Sociedad, Colombia CoLav, Universidad de Antioquia-AmeliCA Métricas, Colombia \\ auribe.bibliotecologia.udea@gmail.com
}

\section{Diego Restrepo-Quintero}

Universidad de Antioquia. Facultad de Ciencias Exactas y Naturales. Grupo de Fenomenología de Interacciones Fundamentales, Colombia CoLav, Universidad de Antioquia - AmeliCA Métricas, Colombia diego.restrepo@udea.edu.co

\section{Jaider Ochoa-Gutierrez}

Universidad de Antioquia. Escuela Interamericana de Bibliotecología. Grupo Información, Conocimiento y Sociedad, Colombia CoLav, Universidad de Antioquia -AmeliCA Métricas, Colombia

jaider.ochoa@gmail.com

\section{César Pallares}

Universidad de Antioquia, Vicerrectoría de Investigación. Unidad de Inteligencia en Investigación, Colombia CoLav, Universidad de Antioquia - AmeliCA Métricas, Colombia cesar.pallares@udea.edu.co

\section{Huber Fernando Gómez-Molina}

Universidad de Antioquia. Vicerrectoría de Docencia. Sistema de Bibliotecas, Colombia CoLav, Universidad de Antioquia AmeliCA Métricas, Colombia

huber.gomez@udea.edu.co

\section{Marcela Suárez-Tamayo}

Universidad de Antioquia. Escuela Interamericana de Bibliotecología. Grupo Información, Conocimiento y Sociedad, Colombia marsumayo@gmail.com

\section{Julián Calle}

Universidad de Antioquia. Facultad de Ciencias Exactas y Naturales. Grupo de Fenomenología de Interacciones Fundamentales, Colombia CoLav, Universidad de Antioquia - AmeliCA Métricas, Colombia julian.callem@udea.edu.co 


\section{Resumen:}

Se presenta una discusión sobre las nuevas métricas para medición de la ciencia y el direccionamiento que debe tomar el sistema en términos de evaluación de la producción científica. Se plantea la diferenciación entre algunas de las propuestas presentadas a la Comisión Europea y los desarrollos de AmeliCA. Se observa la importancia de retomar importantes conceptos que describen el desarrollo de la ciencia, no únicamente desde el punto de vista de la visibilidad alcanzada por diversos medios propuestos por la ciencia abierta, sino también el rescate de aspectos como la creación de comunidades, la formación, la comunicabilidad, entre otros asuntos. Se releva el papel de las métricas especialmente como una herramienta para la toma de decisiones y una forma de rendición de cuentas a la sociedad.

Palabras clave: Métricas responsables, Cienciometría, AmeliCA, Sur Global, Evaluación científica.

\section{Abstract:}

A discussion on the new metrics for the measurement of science and the direction that the system must take in terms of evaluation of scientific production is presented. The differentiation between some of the proposals presented to the European Commission and the AmeliCA developments is proposed. We observe the importance to recognize concepts that describe the development of science, not only from the visibility perspective, including those means derived from open science, but also the recovery of aspects such as the creation of communities, training, communicability, among other issues. The role of the metrics is highlighted especially as a tool for decision making and a form of accountability to society.

KEYWORDS: Responsible metrics, Scientometrics, AmeliCA, Global South, Scientific Assessment.

\section{INTRODUCCIÓN ${ }^{1}$}

El documento Next Generation Metrics de la Comisión Europea (Wilsdon et al., 2017) presenta la ciencia abierta como una innovación que orienta a las comunidades científicas hacia un mundo abierto, con intensificación de relaciones interdisciplinares y transdisciplinares. En sus propuestas para nuevas métricas plantean dos principios básicos: la importancia de la apertura del sistema científico en todos sus niveles, y el mejoramiento del desempeño de grupos de investigación e investigadores orientados hacia la ciencia abierta. El informe introduce el concepto de métricas responsables que permitan la transición hacia la ciencia abierta. Sin embargo, hay que anotar que, con la fundación de sus revistas en el siglo XX, Latinoamérica ya había dado el paso que recién comenzara en Europa con el Plan S (2018). Allí se abre una controversia entre los diferentes conceptos de la orientación futura de la ciencia abierta: mientras el Plan $S$ intenta regular los acuerdos comerciales con las editoriales para pagar por publicar y no por el acceso a las publicaciones, AmeliCA (2019) propone construir infraestructura desde la academia y para la academia (ver también la Declaración de México, LATINDEX-REDALYC-CLACSO-IBICT, 2018).

Así pues, el problema de la ciencia abierta no es únicamente la intensificación de la circulación de información científica de manera gratuita, sino la definición misma de la propiedad de los medios de circulación de la comunicación científica. Esto implica el fortalecimiento de los equipos editoriales académicos para garantizar la apertura y no la negociación del pago de APC.

Si consideramos lo anterior, un concepto de métricas responsables no podría quedarse en los grados de apertura de la ciencia, pues la diversificación de los medios de circulación sólo lleva a la ampliación de las métricas de visibilidad, entendiendo el desempeño de la ciencia como una carrera por obtenerla, y 
desestimando aspectos constitutivos de la generación de conocimiento: la creación de diversidad de formas de comunidad académica, las diferentes posibilidades de alcance en términos territoriales o de generación de relaciones de colaboración intersectoriales, los procesos de formación de nuevos investigadores, y los avances tanto disciplinares como interdisciplinares. Se propone, por tanto, la observación de la diversidad en las formas organizativas de la investigación y los diferentes impactos, no sólo de la apertura sino del desempeño en la creación de conocimiento en el establecimiento de lazos, tal y como lo propone la Declaración de Panamá sobre Ciencia Abierta (2018).

La pregunta interesante es si realmente es posible derivar métricas diferentes de evaluación desde dos iniciativas diferenciadas geográficamente entre norte y sur. Es un hecho que la ciencia no tiene características nacionales y que las regiones del norte y del sur trabajan bajo códigos comunes de construcción de comunidad (Luhmann, 1996). La diferenciación en métricas no admite modelos parciales geográficamente hablando. Esto implica que serían dos visiones métricas viendo al mundo desde perspectivas diferentes. Por un lado, una que centra el desempeño, principalmente en términos de visibilidad y apertura del conocimiento Plan S- y, por otro lado, la que considera, desde el punto de vista de la diversidad organizativa, estrategias distintas de producción y circulación con restricciones a la mercantilización de la producción investigativa AmeliCA-.Al respecto Richard Poynder (2019) subraya la diferencia entre ambas estrategias para abordar el problema del futuro de la ciencia abierta como dos estrategias diferenciadas que tal vez puedan resultar complementarias.

El problema de la responsabilidad de las métricas no está asociado al grado de objetividad que se considere como valor moral del resultado métrico en sí mismo. El problema está en el procedimiento para determinar jerarquías y prestigios. Algunos de estos problemas son los siguientes:

- Las métricas actuales no tienen memoria, personajes ya fallecidos en la sociología que son referentes de campo no logran sobresalir en los índices citacionales, simplemente porque no han publicado en los años recientes y no entran dentro del conteo de un factor de impacto. Si bien la investigación experimental acude a la inmediatez de los últimos descubrimientos, ni la filosofía, ni la filología o el psicoanálisis funcionan así.

- Las métricas actuales tampoco consideran realmente el valor de la prioridad en el descubrimiento, principio fundamental de la ciencia. Las métricas consideran la visibilidad y el impacto, pero no hay inferencia alguna en el valor de la innovación.

- La ciencia es un trabajo colectivo; existen líderes, pero esos líderes no son nada sin sus equipos. Se individualiza la competencia y los procedimientos métricos entran en conflicto cuando se intenta contrastar el valor de una publicación escrita entre más de 5000 autores $^{2}$ con una que no tiene colaboraciones.

- La expansión o retracción de la ciencia se observa desde el punto de vista del crecimiento de la producción, pero no el estado de las comunidades y sus dinámicas particulares de interacción, vigencia de los procesos de formación de nuevos investigadores, etc., que permitan indicar su consolidación o no en las formas de producir conocimiento.

Proponer modelos de métricas responsables implica:

- Un ejercicio cooperativo de evaluación de lo que permite mejorar la observación del desempeño de los mismos científicos. En este sentido la ciencia abierta tiene un papel muy importante: los algoritmos de medición (abiertos en GitHub), los datos (la circulación abierta no comercial de los metadatos) y el diálogo entre los miembros de la comunidad (Blog AmeliCA ${ }^{3}$ ) que se observa en los modelos de medición deben ser abiertos.

- El rescate de data histórica que permita observar la evolución del conocimiento, no sólo desde el punto de vista de las citas más antiguas, sino desde la observación de los procesos de 
institucionalización de la ciencia en el Sur Global. La historia siempre ha sido contada desde Europa. Habría que ver qué polaridades cambian con otros datos y otros relatos.

- Métricas orientadas a la búsqueda de criterios para medir diferentes formas de desempeño y no para encontrar sólo a los más visibles. La utilización de la visibilidad a través del impacto o las descargas o las menciones es insuficiente para observar el cuadro completo de dinámicas.

- Contrastar el desempeño de las prácticas de las revistas universitarias y las revistas comerciales en términos de alcances en la construcción de comunidades. Es importante hacer seguimiento a estas prácticas como posibilidad de generar estrategias para un balance pertinente al desarrollo de la academia sin tener que incurrir en altos costos para poder generar circulación del conocimiento. Esto constituye una dinámica especialmente importante para los países del sur global.

\section{¿Por qué no son SUficientes las Métricas y las métricas alternativas existentes?}

El descontento generalizado en un sector importante de las ciencias respecto a las herramientas de medición de desempeño es el síntoma de transiciones que ha traído el inicio de milenio para las prácticas científicas: la introducción ansiosa de la necesidad de automatización de los procesos de evaluación, la ilusión de alcanzar herramientas objetivas para realizarla, la angustia de la acumulación sin fin de información científica y la presión de los financiadores para entender de una manera fácil y sencilla quiénes son los mejores. Es visible en este contexto el incremento global de la producción de artículos y la explosión de multiplicidad de formas de colaboración científica desde diferentes regiones en el mundo (Dong, Ma, Shen y Wang, 2017; Wagner, 2008).

Producto de esta situación han proliferado múltiples sistemas de indexación de la producción científica que intentan ordenarla. Los criterios de orden han estado anclados a la evaluación de la visibilidad como el factor más importante. Las jerarquías generadas por las medidas de impacto (ver las principales en figura 1) premian la visibilidad de las comunidades con lazos más fuertes, que logran consolidar sus colegios invisibles en grupos transnacionales de investigación científica experimental, especialmente invisibilizando esfuerzos más locales o regionales o de formas de investigación más hermenéuticas, teóricas o de impactos delimitados.

Figura 1. Índices de impacto más usados en la evaluación científica

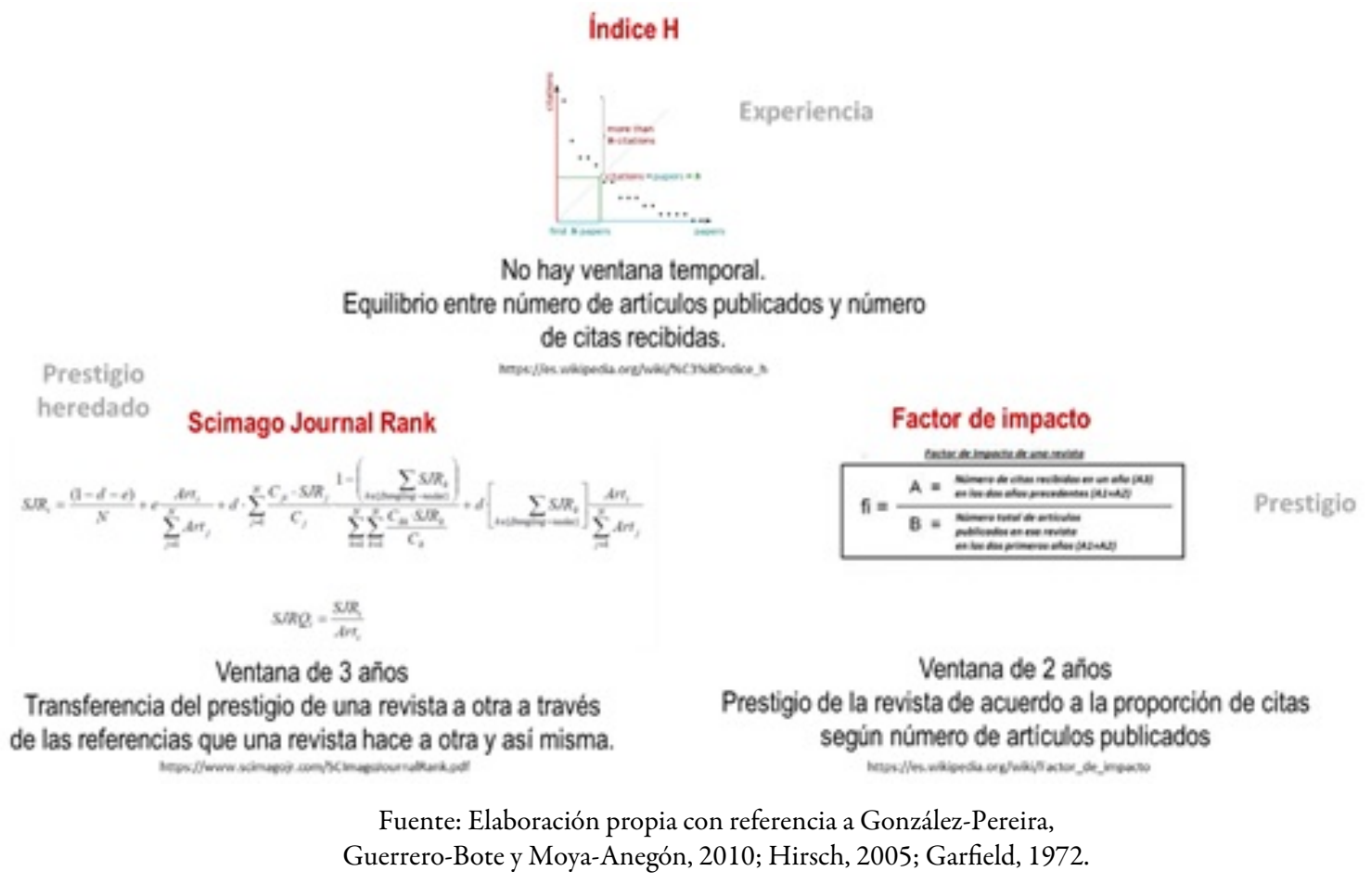


Asimismo, la industria editorial ha capitalizado la propiedad de una parte importante de las revistas científicas de Estados Unidos y Europa. A la vez que, en otras regiones del globo, han proliferado grandes cantidades de publicaciones hechas en la academia para académicos, pero con menos recursos que las revistas de las grandes editoriales como Elsevier, Springer, Taylor \& Francis, entre otras (ver figura 2). De los 5000 editores con publicaciones presentes en Scopus, el 40 \% proviene de 12 casas. Según el Scielo Citation Index en el 2016, de 795 revistas indexadas para ese momento el $89.1 \%$ pertenecían a universidades y asociaciones científicas (Vélez-Cuartas, Lucio-Arias y Leydesdorff, 2016). Esta diferencia también se ve reflejada en las capacidades para hacer visible el conocimiento producido. Mientras las grandes editoriales son visibles en las bases de datos que determinan los indicadores de impacto y la calidad de las revistas - por ejemplo Elsevier, dueña de Scopus-, la gran mayoría de los editores universitarios utilizan recursos de acceso abierto con pocas propuestas para generar indicadores de desempeño de la ciencia. De 26.796 revistas de la base de datos Latindex para América Latina y la península Ibérica, aproximadamente unas $840^{4}$ están en Scopus.

Figura 2. Representación de las casas editoriales en Scopus

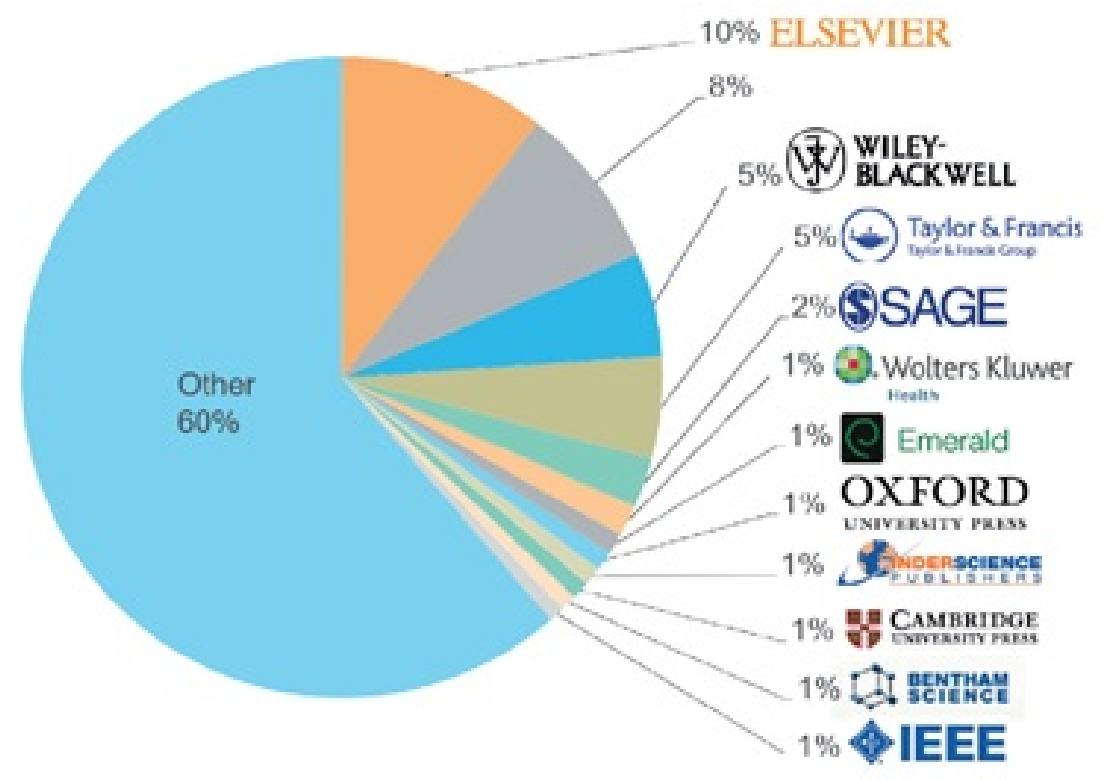

Fuente: Elsevier, 2017

Esta situación ha permeado los mecanismos de valoración del conocimiento, en tanto la producción más válida no está basada en criterios de evaluación de la calidad del trabajo presentado ni en la consolidación de dinámicas organizativas de la ciencia, sino en métricas condicionadas por la cantidad de citas disponibles en la base de datos de referencia que demuestran visibilidad. La visibilidad tradicionalmente se ha considerado como un indicador de prestigio (De Solla Price, 1973). El prestigio en las comunidades científicas es reflejo de reconocimiento de autoridad. La controversia surge cuando las métricas generadas desde algún sistema, sea este Scopus, Web of Science o Google Académico, divergen y generan calificaciones que expresan contradicciones en el sistema, o cuando, de manera especial, un sistema u organización de gestión ciencia privilegia unas sobre otras. Desde ese punto de vista, la visibilidad se hace relativa a la disponibilidad de información para evaluarla según las bases de datos (ver figura 3). 
Figura 3. Divergencias en las métricas para la clasificación de revistas Publindex en Colombia.
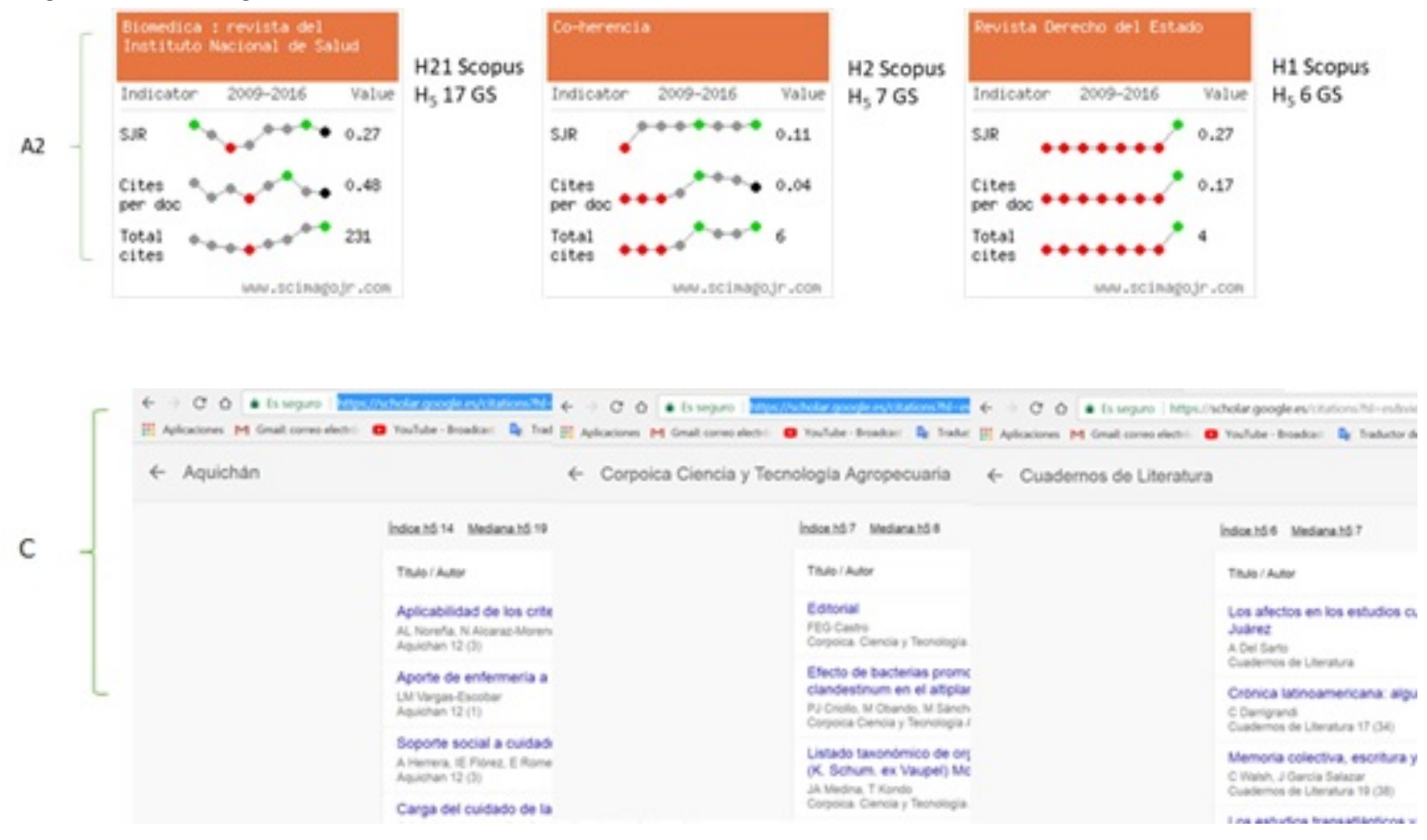

Fuente: Scimago y Google Académico, consultados en junio de 2018.

Sólo para ejemplificar la controversia, si tomamos el Publindex, que es el índice nacional de revistas científicas en Colombia, hay 4 categorías, de mayor a menor: A1, A2, B y C. En el ejemplo de la figura 3 las revistas A2 difieren sustancialmente en el número de citas recuperadas de Scopus, mientras que la revista Biomédica para 2018 tenía 231 citas totales, las otras dos revistas de humanidades tenían 6 y 4 citas totales respectivamente. En contraste, las revistas clasificadas en $\mathrm{B}$ y $\mathrm{C}$ tienen un $\mathrm{H} 5$ de Google Académico mayor o igual que las revistas clasificadas en A2. Estas inconsistencias son derivadas de elegir el prestigio de las bases de datos como criterio de selección para la clasificación y no la visibilidad, o, en el mejor de los casos, la evaluación de calidad de las mismas revistas.

De otro lado, el estudio de Martin-Martin, Orduna-Malea, Thelwall y Delgado López-Cózar (2018) ya expresa la diferencia de disponibilidad de referencias entre Web of Science, Scopus y Google Académico. Su estudio refleja que, en áreas como las humanidades, las artes y las ciencias sociales tienen entre $53 \%$ y 59 $\%$ más de citas disponibles en Google Académico que en los otros sistemas, a diferencia de la química y las ciencias de los materiales, que alcanzan a tener la mayoría de las referencias dentro de los sistemas Web of Science y Scopus. Esto marca una diferencia bastante importante que permite develar la relatividad de los puntos de vista con los que se mide la visibilidad. También expone la necesidad de buscar mayor cantidad de fuentes para integrarlas y lograr así una fotografía más real y desde mejores ángulos.

Así se genera una tensión entre disciplinas y proyectos interdisciplinarios y transdisciplinarios en la disputa por recursos y visibilidad. Como consecuencia de la disputa surge una presión por el reconocimiento de diferentes formas de hacer ciencia, como, por ejemplo, el reclamo de las humanidades y las artes de un estatus como productoras de conocimiento válido (Piñeres Sus, Montes, y Vélez Cuarta, 2017), que también reclaman las formas de conocimiento ancestral desde sus propias construcciones epistemológicas. El reclamo por el reconocimiento señala que las diferentes comunidades disciplinares lo valoran, pero no está siendo observado por métricas limitadas que obvian aspectos como la generación de comunidades, los alcances geográficos en la dispersión del conocimiento, la comunicabilidad en términos tecnológicos y humanos, la interdependencia organizacional, las capacidades de generar innovación y relaciones con entornos no académicos, o la formación de nuevos investigadores. 
Las métricas orientadas sólo por la visibilidad no parecieran expresar el conjunto pleno de dinámicas sociales en la producción de conocimiento. Esto va en detrimento de tener una panorámica general de evolución de las comunidades que permita entender la ciencia en términos de vínculos generados, y no sólo de patrones de crecimiento de la producción y su visibilidad. La ciencia se hace efectiva en la vinculación, y la acumulación de productos es únicamente uno de los efectos de la construcción colectiva. De Solla Price (1973) lo advertía en el paso de una pequeña a una gran ciencia compuesta por equipos de investigadores. Estos equipos en los albores del siglo XXI se articularían a través de redes complejas que estarían determinadas tanto por la interacción como por la expansión de múltiples colegios invisibles en redes de flujos de conocimiento desde sus diferentes formas de producción (Wagner, 2008). La visibilidad en este océano de producción investigativa es amplificada por los índices citacionales y sirve como estrategia en la obtención de capitales simbólicos (Bourdieu, 2000). Pero la amplificación afecta a aquellos que no entran a los circuitos de las revistas mejor indexadas, no sólo a la invisibilidad sino a la credibilidad en sus procesos de validación del conocimiento. Los principios de la medición basados en la visibilidad, frente a estos síntomas de transformación, parecieran demasiado simples para explicar tanto la emergencia de la complejidad en la producción de conocimiento, como así también la caracterización de su multiplicidad y diversidad para observar el desempeño de acuerdo a su naturaleza diversa.

En más detalle, el mecanismo del prestigio permite la detección de autores que son capaces de atraer a más colegas por la cantidad de citas y publicaciones en un período de tiempo de dos o tres años, dependiendo de la aplicación del Factor de Impacto (Garfield, 1972), o del largo de la vida productiva, según el índice H (Hirsh, 2005). El desarrollo de estas métricas ha generado una explosión de propuestas alrededor de la medición de visibilidad. Pero también surge la respuesta de las métricas alternativas a la observación de la visibilidad reducida al círculo cerrado de las citaciones (Uribe-Tirado y Alhuay-Quispe, 2017; Wilsdon et al., 2017). Ya se cuenta con 117 indicadores entre cienciométricos y altmétricos, y con 51 derivados del índice $H$, de acuerdo a la "tabla periódica" de indicadores del grupo de investigación EC3 metrics (2018). Este estado del arte bien puede simplificarse en diferentes variantes del prestigio que podrían ser los componentes de un indicador sintético.

No obstante, pese a esta elevada cantidad de indicadores cienciométricos y altmétricos, la mayoría de los esquemas de evaluación de los científicos y de la asignación de recursos para proyectos siguen basándose en una sola métrica; ignorando la diversidad de indicadores y las distintas maneras de organización científica que puede tomar una comunidad. Ejemplo de ello es el esquema de medición de grupos de Colciencias, o los indicadores que utilizan los rankings universitarios como el QS. Esta situación ya ha generado rechazo por la comunidad científica y es uno de los sustentos de la Declaración de San Francisco (DORA) y el Manifiesto de Leiden.

No hay duda de que la visibilidad medida por métricas tradicionales y alternativas refleja los logros de la ciencia: el proyecto del genoma humano, la búsqueda de la cura contra el Alzheimer, la red de laboratorios alrededor de la Organización Europea para la Investigación Nuclear (CERN), entre muchos otros. Son casos exitosos, cuyos investigadores líderes de equipos han alcanzado altos índices de visibilidad e impacto. No hay duda en ello, sin embargo, queda la pregunta por el resto, por lo caótico, lo no ordenable en colegios invisibles trasnacionales. Wagner intenta resolver la duda con algunas estadísticas que hablan de baja actividad: "South America is the least common destination for foreign students (hosting only 0.4 percent of the global foreign student population), followed by Africa (1.2 percent)" (Wagner, 2008, p. 67). Una posible interpretación para convertirla en respuesta es que, en realidad, no es muy relevante observar dinámicas científicas en geografías que no logran la acumulación o masa crítica idónea.

Se podría argumentar que la exclusión de revistas dependería directamente de la evaluación de su visibilidad. Según una creencia difundida, son mejores las investigaciones y los artículos de aquellas revistas que tienen un mayor factor de impacto o una mejor reputación. Chavarro, Ràfols y Tang (2018) desarrollaron una investigación para demostrar que la inclusión de revistas latinoamericanas en Web of 
Science y aquellas excluidas no necesariamente responde a criterios universales de la ciencia, como altos estándares editoriales, un alto índice h o la edad de las revistas, sino a otros más particulares, como el país de publicación, la lengua o la disciplina, el producto interno bruto, el tipo de organización o publicación. Aclaran que el índice h es un buen predictor de inclusión, pero al momento de escoger una u otra con un índice h similar, las particularidades pesan más que las características universales. Unas revistas son "más iguales que otras" aunque tengan el mismo impacto, dicen Chavarro Ràfols y Tang (2018). Asimismo, comprueban que en realidad la adscripción a Web of Science muestra más un indicador de pertenencia a una comunidad específica -y una garantía de lectura entre los miembros de esa comunidad- que un indicador de calidad.

Los datos muestran el desbalance para poder construir indicadores que den cuenta de las dinámicas generales de la producción académica. Si sólo se consideran las dinámicas de indexación, Latindex tiene registradas 26.763 revistas en su catálogo iberoamericano, de ésas, 8.846 están en línea. De éstas, Redalyc alberga 1.302 en su portal y Scielo anuncia 1.285, aunque sus datos bibliométricos arrojan un total de 1.448. De éstas, el portal de Scielo Citation Index de Web of Science recupera 1.426 (consultas 9 de Marzo de 2019). Estos números se estrechan aún más cuando se consulta el JCR de 2018 con datos del 2017, donde se encuentran un total de 270 revistas Latinoamericanas (Chavarro, Ràfols y Tang, 2018). Scopus considera 842 , un poco más de 3 veces la cantidad de las indexadas por Web of Science. En total son aproximadamente 1.954 revistas, una vez hecha la cuenta de las revistas únicas y las traslapadas en distintas bases de datos. Si a esto agregamos que las métricas alternativas operan principalmente con los DOI de los documentos y el impacto se mide con las citas disponibles, tenemos no sólo un problema de inobservancia de otros criterios de desempeño, sino también de invisibilidad por la dispersión de información de diferentes bases de datos.

Toda esta situación propone retos en la organización de la información, no sólo para las publicaciones más visibles, sino también para aquellas que no alcanzan los circuitos de circulación que permiten las grandes bases de datos. El trabajo de construcción de métricas responsables requiere un cambio fundamental en la apertura de los metadatos y datos de las investigaciones, en concordancia con lo planteado por el informe Next Generation Metrics, pero también la construcción de criterios que incluyan la diversidad del desempeño de las comunidades en términos de la evolución de su construcción e integración de nuevos actores, no sólo el uso de la información.

\section{Propuestas para un batería de métricas responsables}

Hay tres aspectos fundamentales que explican las dinámicas de la generación de conocimiento basado en criterios científicos y humanísticos. Merton (1973) propuso la existencia de un ethos particular para la ciencia: algunos de sus valores son la universalidad en la generación de tesis, el escepticismo organizado en la valoración de la evaluación como mecanismo de validación, y el comunitarismo como principio de construcción colectiva que respeta a su vez la prioridad en los descubrimientos. Bourdieu (2000) aportó a la discusión el concepto de campo, como un conjunto de prácticas que entran en franca lucha por la adquisición de recursos materiales y simbólicos para obtener prestigio. En este sentido, los valores del ethos no orientarían a una comunidad en consenso, sino a grupos de poder que intentan jugar bajo reglas de competencia por la verdad. Finalmente, Wagner (2008) plantea en los nuevos colegios invisibles una lógica de autoorganización orientada por estructuras que crecen de manera exponencial y que permiten identificar claramente un centro y una periferia.

Ethos, campo y autoorganización son tres principios que permiten observar las dinámicas de crecimiento y diversificación de las diferentes formas organizativas. Los tres principios valoran el prestigio como factor orientador de las comunidades científicas, pero hay diferencias fundamentales en los procesos que llevan al resultado. Desde el punto de vista de Merton (1973), el prestigio se alcanzaría a través del reconocimiento de la prioridad de descubrimiento. Esto nos lleva a plantear el problema de la innovación teórica y metodológica en el hallazgo de nuevas formas de explicar el universo y sus particularidades. Pero a esto habría que añadir la 
valoración de la disponibilidad de capitales en la agrupación de colectivos de investigadores propuestos por Bourdieu (2000) y Wagner (2008). Desde el punto de vista de Wagner, los recursos materiales organizan las estructuras de colaboración alrededor de la localización geográfica de éstos. Desde el punto de vista de Bourdieu, son básicos los recursos relacionales y simbólicos como el capital social construido y la validez de los resultados consensuados por la comunidad.

La introducción de conceptos sociológicos más complejos a la construcción de nuevas métricas permitiría una ampliación de los análisis de la información disponible. En este sentido, empiezan a transformarse las formas de observación de los datos disponibles. Pero para dar este paso habría que identificar claramente los actores que disputan estos recursos. En el caso de la investigación y el entorno actual podrían ser cuatro básicos que constituyen el núcleo de la producción de conocimiento científico y académico: los investigadores, las instituciones, las editoriales comerciales y académicas representadas en sus publicaciones, y las entidades gubernamentales de financiamiento de la ciencia. Desde un punto de vista Luhmanniano (Luhmann, 1996), las acciones de estos actores están orientadas por las comunicaciones que circulan en su entorno. Desde el punto de vista científico, serían todos los productos derivados de investigación posibles (Vélez-Cuartas, Gómez-Flórez, Úsuga-Ciro y Vélez-Trujillo, 2014): artículos, libros, blogs, talleres, cartillas, etc. De acuerdo con la forma misma de las comunicaciones científicas, sería posible rastrear en una estructura robusta las estrategias utilizadas por estos actores para incrementar sus capitales, pero también las innovaciones introducidas en distintos campos de conocimiento (Wu, Wangy Evans, 2019). Esto implica la diversificación de las formas de cooperación e inclusión de nuevos actores que permitan sustentar la producción y la valoración de sus aportes.

De otro lado, es importante considerar que las métricas son herramientas para la evaluación y no las únicas formas de observar el desempeño. Si retomamos las declaraciones de DORA (2012) y Leiden (Hicks, Wouters, Waltman, de Rijcke y Rafols, 2015), las métricas deben ser diversas, pero también deben estar acompañadas de evaluación cualitativa. En ese sentido, las métricas son herramientas para evaluadores que consultan otros criterios de acuerdo con sus intenciones. La construcción de rankings simplifica la riqueza de las dinámicas científicas. En un entorno de Big Data, los más visibles opacan importantes dinámicas emergentes diversas. En este sentido, las métricas responsables construirían marcos comparativos de desempeño en experticias desarrolladas por los tres tipos de actores, en su competencia por la obtención de capitales.

Se pueden identificar algunas orientaciones en la construcción de métricas responsables desde el punto de vista expuesto. En conjunto generarían escenarios evaluativos para pares e instituciones en la identificación de experticias y evoluciones de las dinámicas científicas:

\section{Construcción de comunidad}

En una lógica de construcción de capital social, las citaciones se convierten, no en indicadores de impacto sino de participación en comunidades en expansión o retracción. Ya Garfield había planteado la importancia de este asunto en la detección de lo que llamó historiografiaalgoritmica(Garfield, 2001). Desde el punto de vista de la trayectoria de un tema de la ciencia, a través de las genealogías en las citas se podría reconstruir la historia académica de un conjunto de autores (Lucio Arias y Leydesdorff, 2008). Desde otro punto de vista, estas comunidades observadas - desde la multiplicidad de estrategias que pueden emerger de la complejidad organizativa de la ciencia- tienen necesariamente referentes localizados geográficamente, y pueden observarse estrategias de expansión en la constitución de comunidades, lo que puede considerarse un indicador de los diferentes niveles de desarrollo de la comunidad.

Desde el punto de vista de los autores, su comunidad de citación y referenciación adquiere diferentes densidades y alcances geográficos que pueden ser contrastables (figura 4). Asimismo, las revistas pueden o no generar comunidades de discusión alrededor de los temas que abordan en distintas dimensiones y formas de 
dispersión geográfica (figura 5). Las instituciones, a su vez, van generando experticias que permiten observar su introducción en colegios invisibles temáticos de diferentes impactos.

Figura 4. Comunidades de citación de dos autores. Densidad y grado promedio
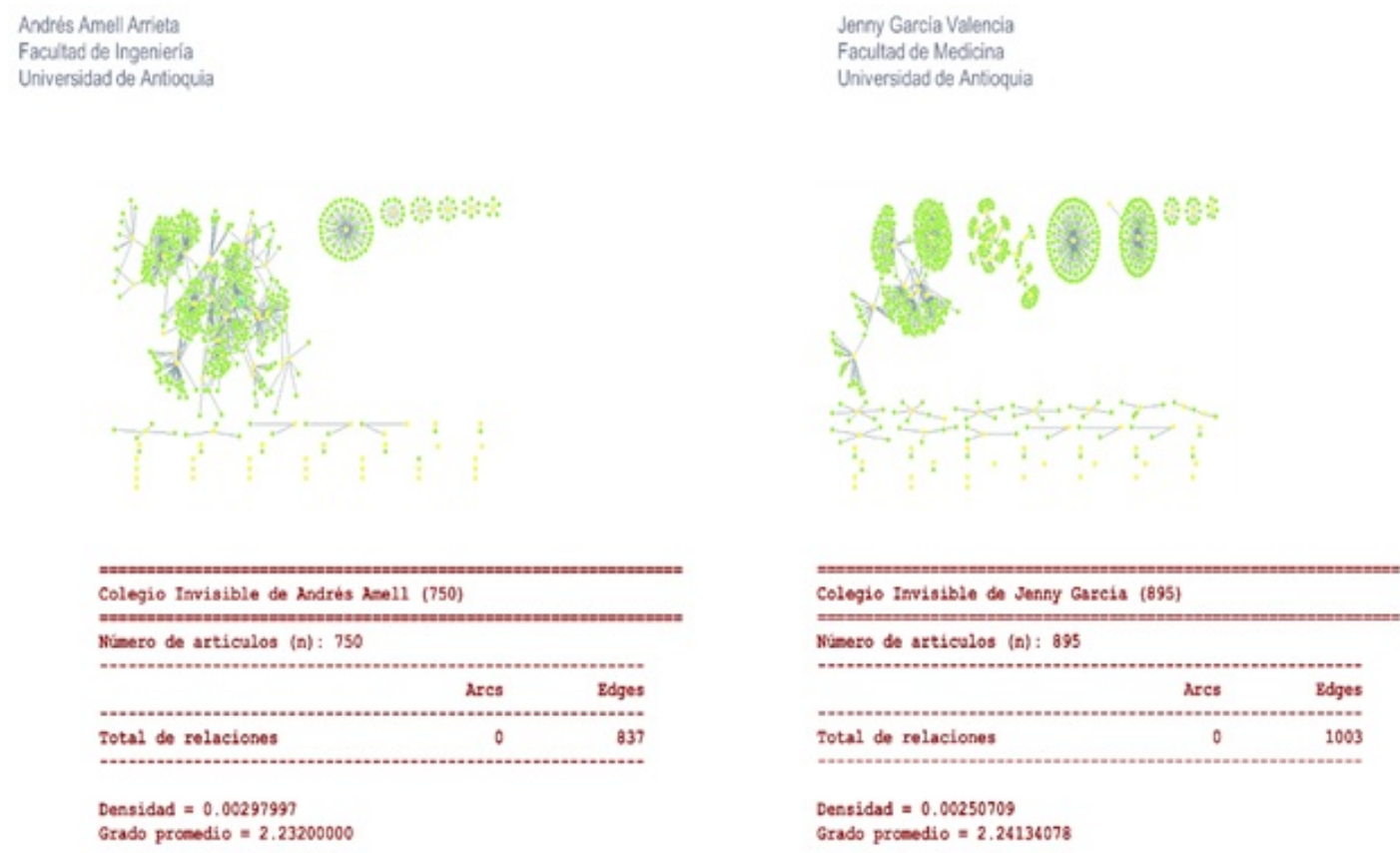

Densidad $=0.00250709$

Grado promedio $=2.24134078$

Fuente: Elaboración propia con la colaboración de Marcela Suárez Tamayo. Datos Google Académico.

Figura 5. Comunidades de colaboración y citación de la Revista de Estudios Politicos de la Universidad de Antioquia. Densidad y grado promedio

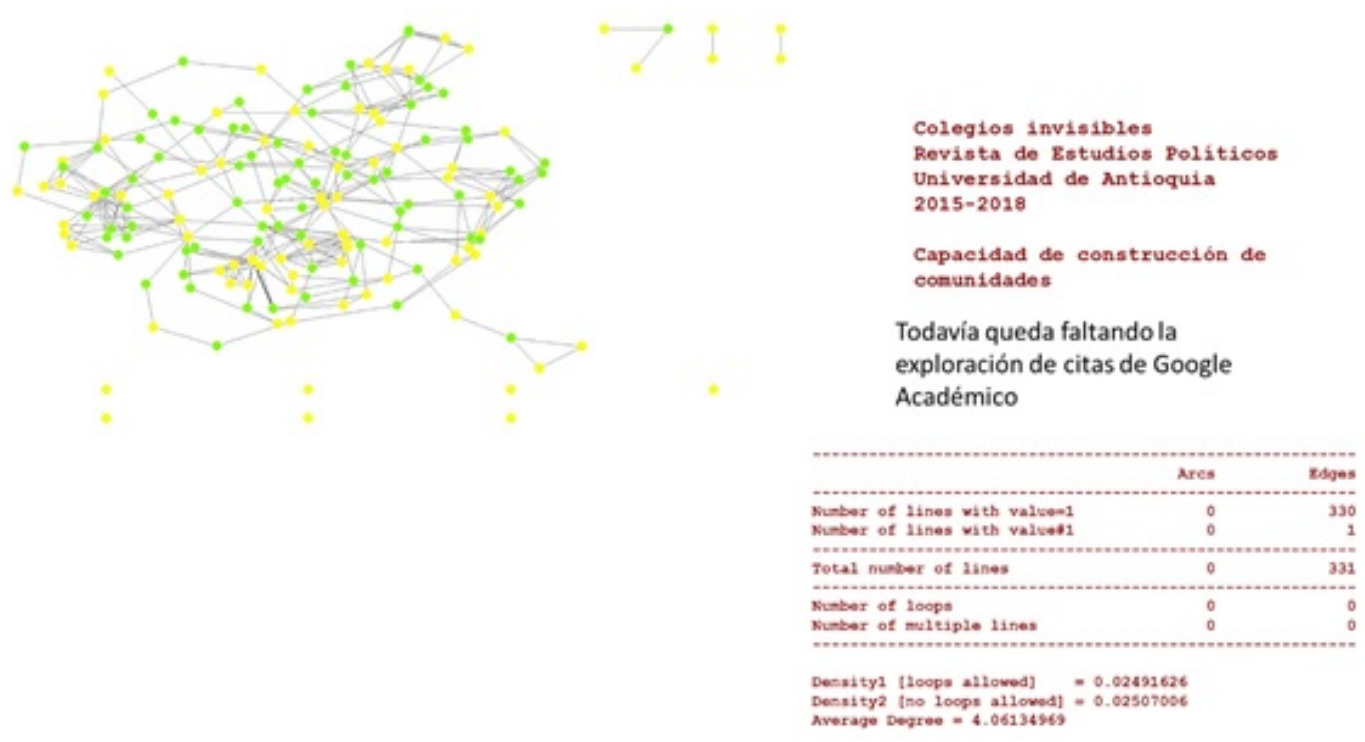

Fuente: Elaboración propia con la colaboración de Marcela Suárez Tamayo. Datos Google Académico.

El alcance de estas comunidades estaría determinado por la dispersión geográfica de las comunidades de referencia (Suárez-Tamayo, Collazo-Reyes y Pérez-Angón, 2018). La figura 6 muestra los alcances de los 
autores de la figura 4. Esta dispersión puede permitir la caracterización de los alcances de la comunidad de los autores en términos de distribución geográfica. El establecimiento de umbrales de la procedencia de citas puede permitir este tipo de observaciones.

Figura 6. Alcance geográfico de las comunidades de citación de dos autores de la Universidad de Antioquia
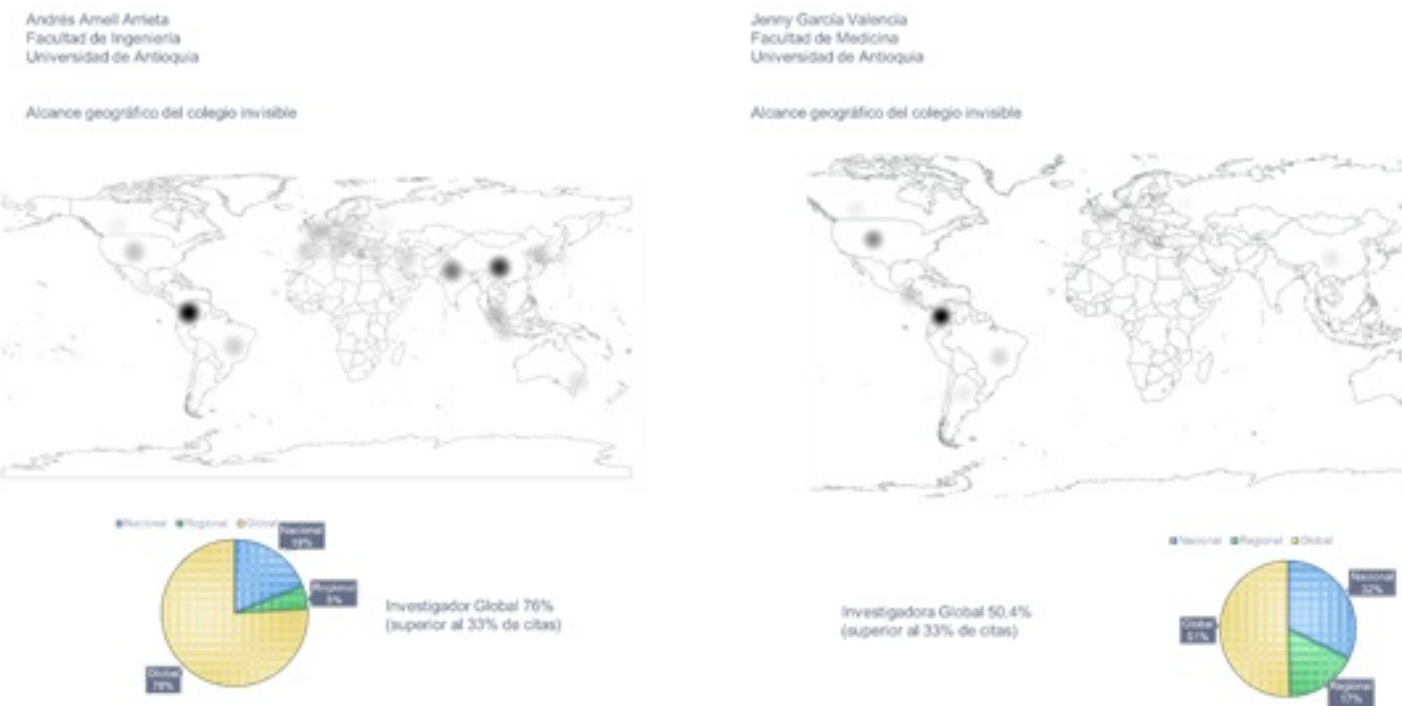

Fuente: Elaboración propia con la colaboración de Marcela Suárez Tamayo. Datos Google Académico.

Desde el punto de vista institucional, tanto la experticia como la consistencia ganada en la producción científica sobre ciertos temas pueden observarse en la continuidad de la producción académica a través del análisis del acoplamiento bibliográfico. La distribución de sus colaboraciones institucionales en el mundo permite la observación del alcance de la comunidad temática formada institucionalmente (figura 7).

Figura 7. Comunidades temáticas formadas institucionalmente. Estructura de colaboración, alcances geográficos y trabajo interdisciplinar sobre investigación celular en la Universidad de Antioquia

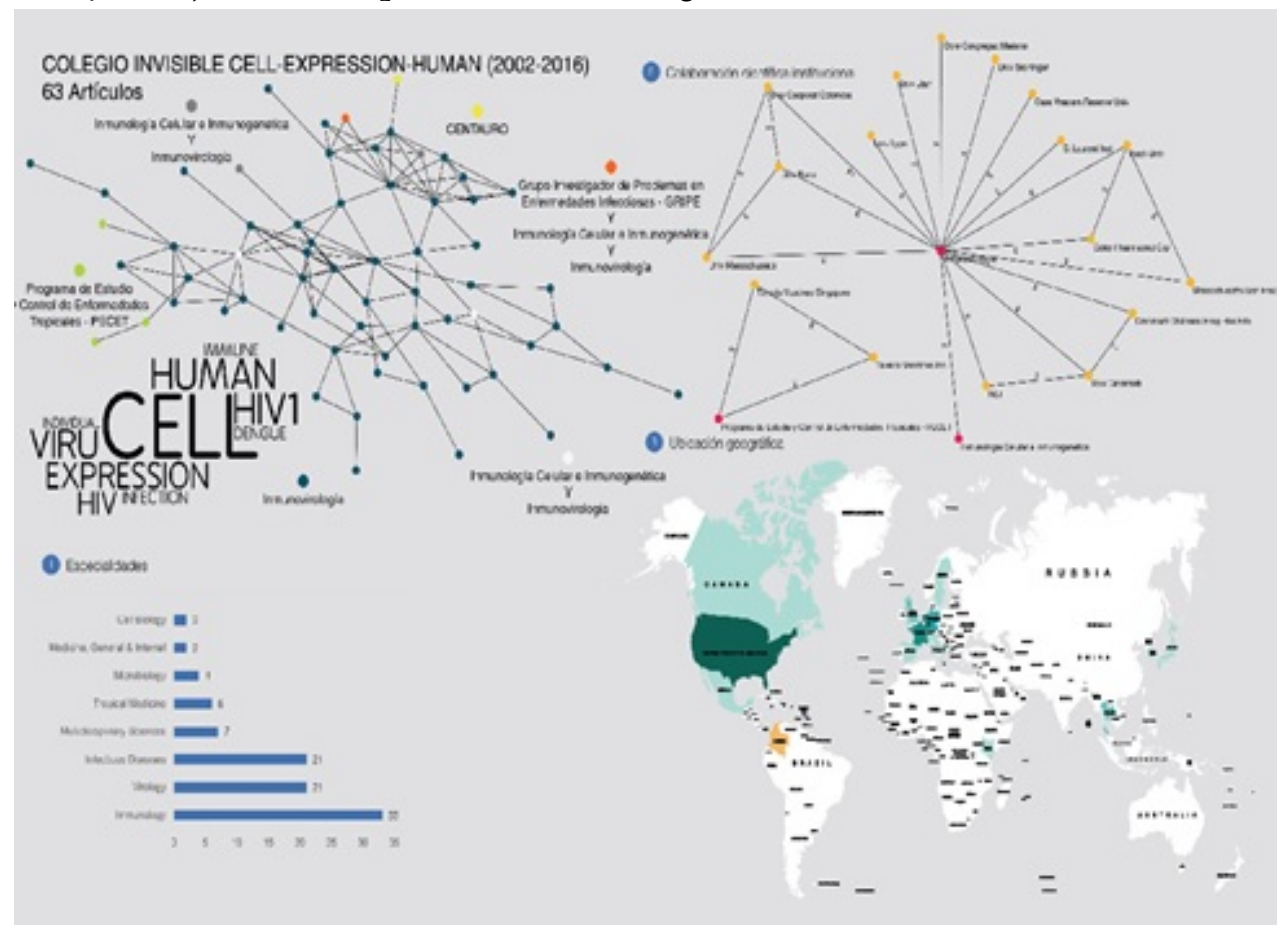

Fuente: Vélez Cuartas et al.2017. 


\section{Redes de conocimiento}

Las redes de conocimiento son formaciones interorganizacionales de circulación y difusión de conocimiento (Casas, 2001). La producción científica genera diferentes estructuras con diferentes alcances. Para el caso de los investigadores de la figura 4, es posible hacer una caracterización también en su capacidad de generar relaciones intersectoriales y la intensificación de su colaboración a partir de coautorías, lo que podría redundar en la ampliación de conocimiento científico o la posibilidad de generar innovaciones en distintos sectores sociales (figura 8). Por ejemplo, la coautoría de artículos entre organizaciones académicas y civiles o gubernamentales puede indicar procesos de construcción colectiva de conocimiento para el mejoramiento de políticas públicas, o la participación en procesos de movilización social para la comprensión de fenómenos, o la introducción de innovaciones sociales o transformativas.

Figura 8. Redes de conocimiento interorganizacionales y distribución sectorial en la generación de productos investigativos

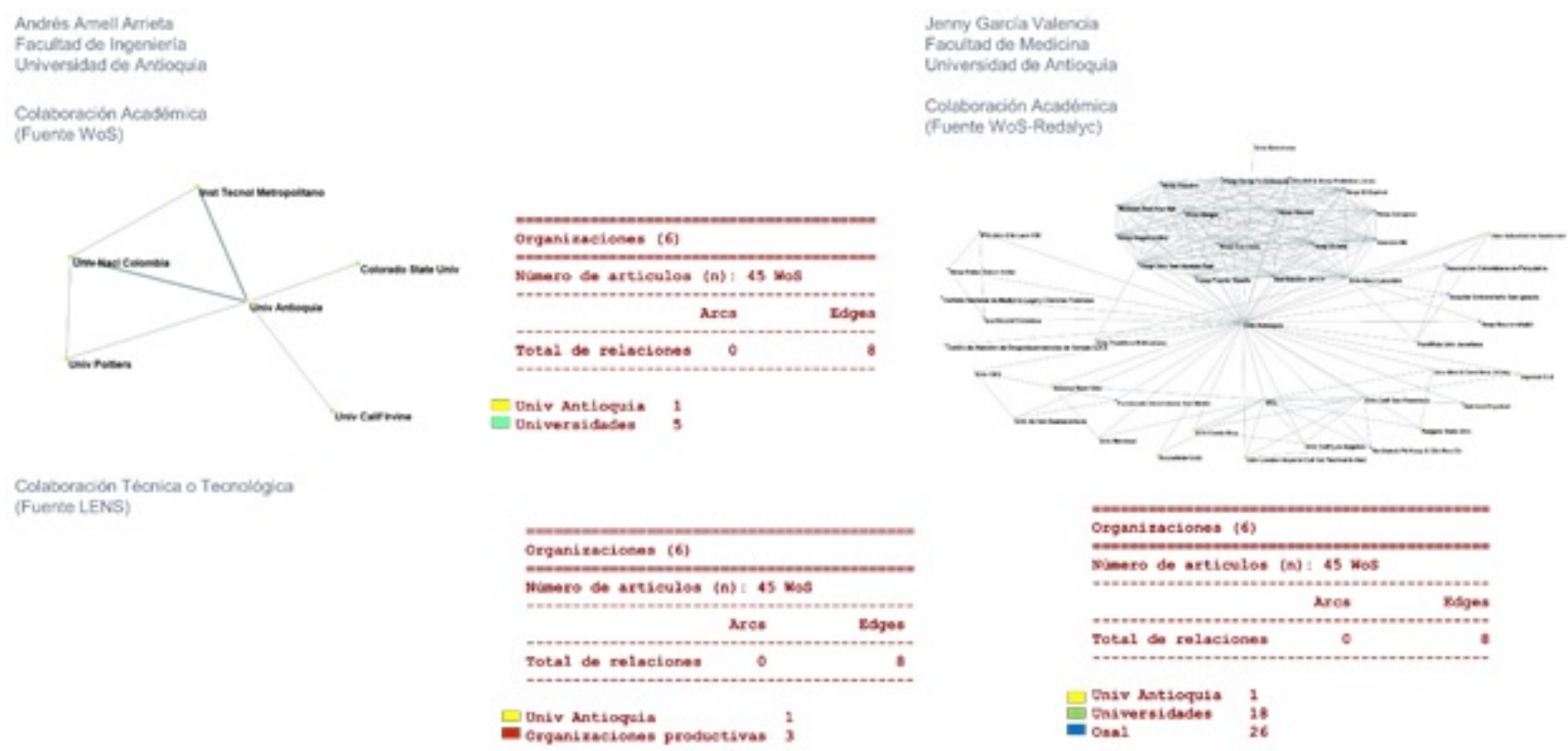

Fuente: Elaboración propia con la colaboración de Marcela Suárez Tamayo. Datos Google Académico.

\section{Distribución de las publicaciones en bases de datos}

La identificación de la distribución de los artículos en diferentes bases de datos permite generar una perspectiva que observa las estrategias de difusión y visibilidad de la información de investigadores e instituciones en su producción (figura 9 y figura 10). La distribución relativa de publicaciones puede dar una idea importante a los diferentes actores de sus estrategias para mejorar la comunicación con sus comunidades de referencia. La distribución en bases de datos, contrastada con los alcances de sus comunidades y redes de conocimiento, permite generar un panorama más amplio de desempeño. 
Andress Amell Arrieta Facultad de ingenienis Universidad de Antioguia

Articulos identificades 71

Citas WoS: 379

Citas RG: 425

Figura 9. Distribución de la producción de la Universidad de Antioquia en 4 bases de datos para el año 2016
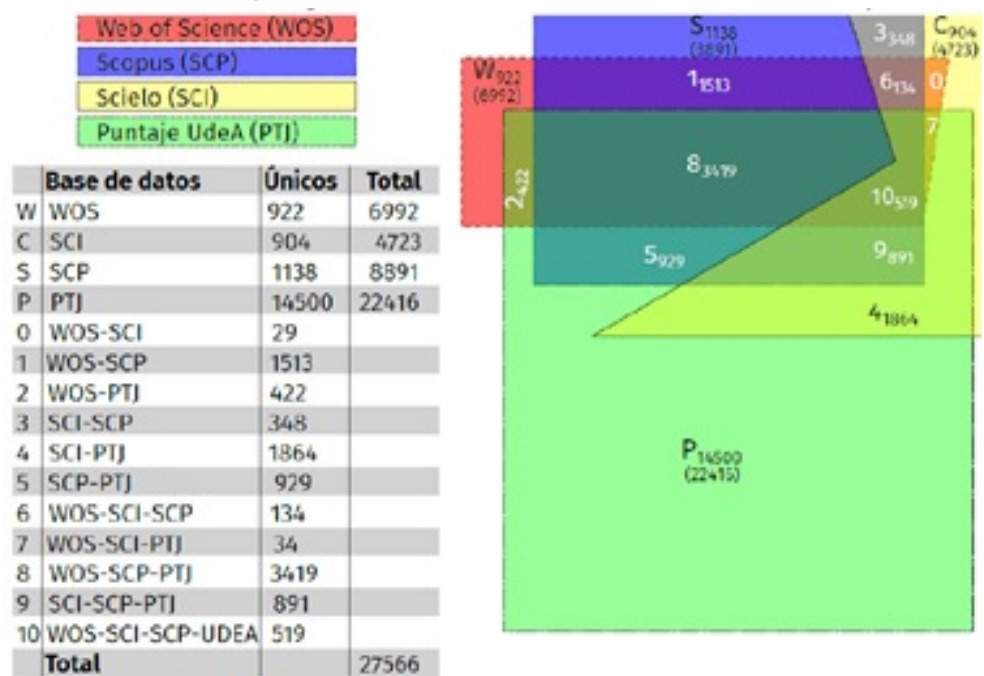

Fuente: Vélez-Cuartas et al. 2017.

Figura 10. Distribución de la producción de dos investigadores en Redalyc, Web of Science, Scopus y Scielo Citation Index

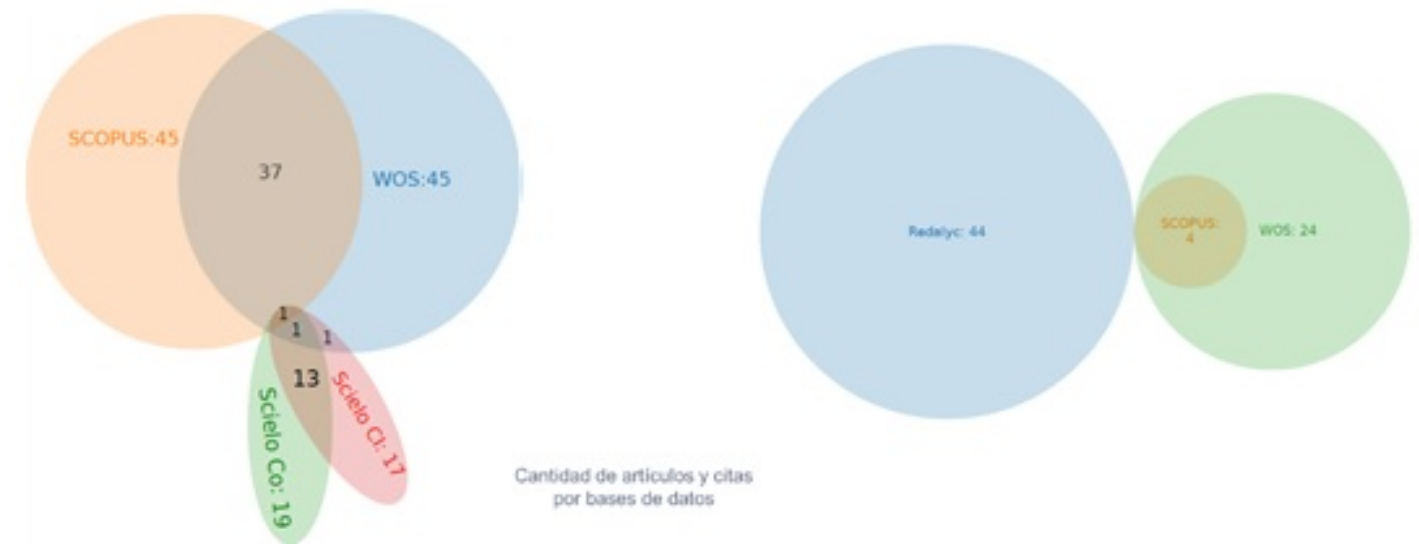

Fuente: Elaboración propia con la ayuda de Marcela Suárez Tamayo y Diego Restrepo. Datos de las bases de referencia.

\section{Evolución de los colegios invisibles en el Sur Global}

De un lado estaría la reconstrucción de la institucionalidad científica, a partir de la construcción de indicadores geohistoriométricos que lleven a otros relatos de la institucionalización de la ciencia (FloresVargas, Vitar-Sandoval, Gutiérrez-Maya, Collazo-Rodríguez y Collazo-Reyes, 2018) a través de la recuperación de metadatos históricos. A ello se dedica, por ejemplo, el proyecto del Atlas Histórico de la Ciencia Latinoamericana y del Caribe. 
Del otro lado estaría la identificación de los autores y los grupos de trabajo más relevantes, independientemente del tiempo en que escribieron y su vigencia en las prácticas de citación. El equipo de webometrics ha adelantado un trabajo importante en este sentido (http://www.webometrics.info/en/hlarg erthan 100). Es importante disipar la ilusión de prestigios relativos y cambiarlo por la figura de comunidades consolidadas para la circulación del conocimiento. Los grandes líderes de campo son científicos y académicos que trascienden la temporalidad y los factores de impacto, como se dijo, desconocen esta situación.

\section{Apuesta por el acceso abierto y niveles}

La identificación de las diferentes vías del acceso abierto, con sus aspectos positivos y negativos, y especialmente la identificación del Openness Index (Nichols y Twidale, 2017) a nivel institucional, de facultad, grupo y hasta por investigador es un aspecto fundamental, para saber en la realidad, a nivel cuantitativo y comparable, qué tanta apuesta estamos haciendo por el acceso abierto y hacia qué niveles, hacia qué tipo de comunicación científica, entre otras cuestiones, se está apuntando (Uribe-Tirado et al., 2019). A su vez, de manera específica en esos niveles, identificar un aspecto crítico actual que, como se indicó, está diferenciando propuestas, como el Plan $S$ europeo y AmeliCA del sur global, respecto a la cuantificación del pago por publicar APC o el pago por suscripciones a bases de datos comerciales y oligopólicas (Luchilo, 2019) que conllevan altos costos para los países, organismos de ciencia, universidades y demás, no siempre transparentes (https://reemaps.intact-project.org). Por ello, esos datos son de suma importancia para entender los costos, en contraste con los resultados, en términos de construcción de comunidades de la producción de distintos autores e instituciones.

\section{La visibilidad en medios de comunicación y diferentes plataformas académicas-sociales}

Uribe-Tirado, Ochoa Gutiérrez y Medina Alfonso (2019) proponen un método de rastreo de los autores en medios de comunicación agregados por Google News. Este método permite observar la visibilidad de los investigadores en las agendas de los medios de comunicación, ante la necesidad de ampliar la concepción de visibilidad e impacto y no sólo centrarla en los pares científicos, sino en otros agentes de la sociedad, como medios de comunicación, políticos, profesionales, comunidades, etc., tal como lo reiteran Bastow, Dunleavy y Tinkler (2014) al presentar sus tres niveles: tradicional academic, mediating middle y external society, los cuales responden a la perspectiva de vinculación con el entorno, que es la base del proyecto que soporta este trabajo. Igualmente, el uso de métodos relacionados con métricas alternativas (Uribe-Tirado, Ochoa Gutiérrez, Ruiz-Muñez y Fajardo-Bermúdez, 2019) permitiría observar el desempeño de la comunicación científica de acuerdo con la capacidad de uso de diferentes plataformas académicas-sociales (ResearchGate, Mendeley, Twitter, Facebook, etc.).

Estas plataformas que amplían la visión de visibilidad e impacto en relación con diferentes agentes de la sociedad, permiten identificar la capacidad de difusión y las estrategias de comunicación generadas por los científicos para ganar prestigio, es decir, permitiría una evaluación de la actividad comunicativa de los investigadores, $y$, por tanto, identificar la relación positiva (correlación) entre visibilidad social y académica, impactos sociales y académicos, como lo evidencian distintos estudios desde la perspectiva de altmetrics (https://universoabierto.org/tag/altmetrics/). Algunos indicadores propios han sido ensayados por Uribe-Tirado, Vallejo Echavarria y Betancur Marín (2016) al respecto, como el D/T Metrics, que observa las descargas respecto a su distribución temporal. Además, es necesario ir construyendo otros para no quedar sujetos de nuevo solamente a determinadas empresas, en este caso las de altmetrics: Altmetrics.com, ImpactStory, Plumx, Kudos, etc., sino que sean complementarias. 


\section{Lengua de publicación}

La comunidad lingüística de referencia es un indicador que poco a poco va a transformarse por los traductores automáticos que seguramente tendrán sus desarrollos más importantes en la próxima década. Sin embargo, las particularidades idiomáticas y la selección de lenguas en las que publican las revistas aún son indicador de orientación de la comunicación hacia comunidades específicas. El contraste de las lenguas elegidas para la publicación y las comunidades detectadas puede permitir entender el alcance de los trabajos de investigadores, instituciones y revistas.

\section{Formación de nuevos investigadores}

Es posible identificar los trabajos de grado y tesis de posgrado en los que son citados los artículos de investigación de diferentes autores. Esto implica la participación en los procesos de formación de los estudiantes en la utilización del conocimiento para el fin formativo. En la figura 11 se muestra el desempeño de dos investigadores respecto al número de trabajos dirigidos en distintos niveles de formación, y las citas recibidas por trabajos de grado y tesis distribuidos geográficamente. La distribución geográfica muestra también el alcance del apoyo en la formación a nuevos investigadores por parte de las publicaciones de estos autores.

Figura 11. Participación de dos autores en procesos de formación de investigadores como tutorías y citas en trabajos de grado y tesis

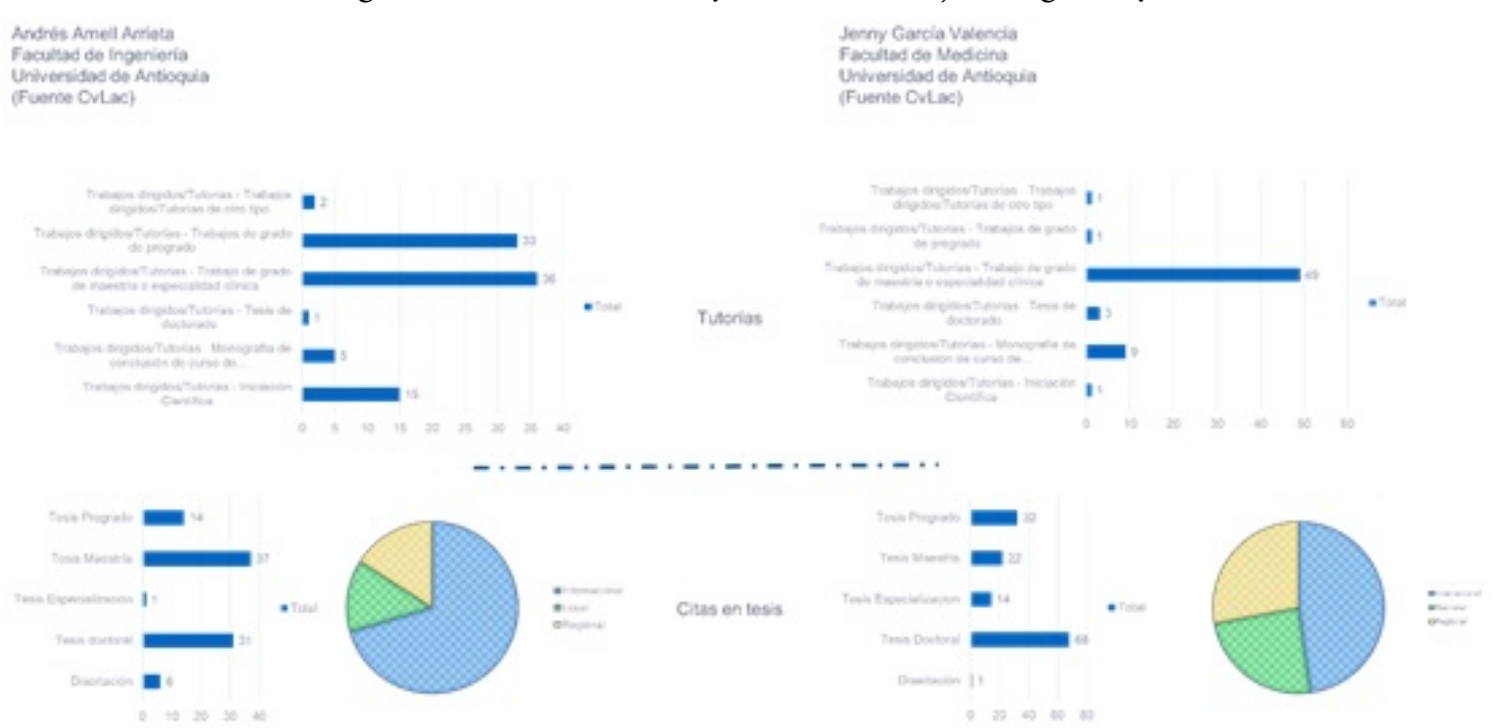

Fuente: Elaboración propia. Datos de CvLac (Colciencias) y Google Académico.

En síntesis, respecto a estas 8 orientaciones es necesario indicar que no son las únicas métricas posibles, pero en un trabajo juicioso sería posible derivar de los estudios sociales de la ciencia y de los mecanismos colectivos de diálogo, una batería de métricas dinámica que permitiera observar la evolución de la ciencia, no únicamente desde el punto de vista de la visibilidad y el impacto - que ya se ha dicho que es un punto de vista sesgado (un ángulo)-, ya que es necesario relacionarlos con un punto de vista más amplio, la vinculación.

El trabajo de AmeliCA métricas busca, entonces, avanzar en mecanismos responsables que permitan también expresar las dinámicas científicas del Sur Global, no únicamente desde el punto de vista de la visibilidad y el impacto. 


\section{Conclusiones}

Tras todo lo anterior, podemos avanzar en algunas conclusiones, que es necesario seguir construyendo:

- Hay una nueva transformación de las formas organizativas e institucionales de la ciencia, afectadas especialmente por el movimiento hacia la apertura en todas las etapas y estadios de la producción y circulación del conocimiento. Estas transformaciones generan propuestas diferentes. Aquí se contrastaron las posturas del Plan S y AmeliCA desde el punto de vista de los principios para la construcción de métricas. Se evidencian diferencias importantes en los propósitos de observación y las diferentes comprensiones sobre el concepto de responsabilidad.

- Se ha hecho un repaso importante por algunos de los problemas fundamentales de las métricas y las métricas alternativas para tratar de definir el horizonte y las dinámicas de la producción científica. Básicamente, el camino de la visibilidad e impacto, como principios para la construcción de nuevos modelos de métricas, no logra recoger la diversidad y complejidad de las dinámicas globales de la ciencia en redes imbricadas entre el norte y el sur y desde el sur global.

- Se propusieron algunos ejemplos que permiten dar cuenta del concepto de métricas responsables, no desde el modelo matemático, pues la responsabilidad es una valoración moral y no estadística. Esta valoración moral sólo puede confirmarse en tanto exista una comunidad que permita la calificación y la participación en algún grado en su construcción, aplicación y reflexión. La propuesta de AmeliCA métricas en este sentido es generar mecanismos abiertos en los procesos de construcción de los propios indicadores. Una vez se lancen las primeras propuestas se requerirá de la concurrencia de la comunidad cienciométrica (unida a la bibliométrica y altmétrica) ${ }^{5}$ del sur global, para perfeccionar y potenciar modelos de evaluación pertinentes desde diferentes ángulos, métricas (cualitativas, cuantitativas, mixtas; tradicionales y recientes, etc.).

- Al construirse como un proyecto cooperativo interinstitucional, se propone un cambio en las formas de autoobservación y evaluación desde la base de la producción científica. Esto implica una fuerte presión sobre las políticas públicas de los distintos países. Implica la generación de modelos que sirvan a los gobiernos del sur global para poder ordenar sus prioridades en cuanto al diseño de instrumentos de política. Se espera con esta propuesta que se pueda mejorar en pertinencia y asertividad al momento de orientar la ciencia y las humanidades en la transformación.

\section{ReFERENCIAS}

AmeliCA Conocimiento Abierto (2019). AmeliCA vs Plan S: Same target, two different strategies to achieve Open Access [Canal de Youtube]. Recuperado de https://www.youtube.com/watch?v=5kXe1UO4beE

Bastow, S., Dunleavy, P., y Tinkler, J. (2014). The impact of the social sciences: How academics and their research make a difference. UK: Sage.

Bourdieu, P. (2000). El campo científico. En Los usos sociales de la ciencia (pp. 11-27). Buenos Aires: Nueva Visión.

Casas, R. (2001). La formación de redes de conocimiento: una perspectiva regional desde México. Barcelona: Instituto de Investigaciones Sociales de la UNAM/Anthropos.

Chavarro, D., Rałfols, I., y Tang, P. (2018). To what extent is inclusion in the Web of Science an indicator of journal 'quality'? Research Evaluation, 27(2), 106-118. https://doi.org/10.1093/reseval/rvy001

De Solla Price, D. (1973). Hacia una ciencia de la ciencia. Barcelona: Ariel.

Declaración de Panamá sobre Ciencia Abierta (2018). Recuperado de https://karisma.org.co/DeclaracionDePanama

Declaración de San Francisco de la Evaluación de la Investigación [DORA] (2012). Recuperado de http://blogs.ujaen .es/cienciabuja/wp-content/uploads/2013/10/dora.pdf 
Dong, Y., Ma, H., Shen, Z., y Wang, K. (2017). A Century of Science: Globalization of Scientific Collaborations, Citations, and Innovations. Recuperado de http://arxiv.org/abs/1704.05150

EC3 Metrics (2018). Periodic Table of Scientometric Indicators. El profesional de la Información. Recuperado de ht tp://www.elprofesionaldelainformacion.com/notas/wp-content/uploads/2018/06/tablaper3.pdf

Elsevier (2017). Scopus Content Coverage Guide. Recuperado de https://www.elsevier.com/solutions/scopus/content Flores-Vargas, X., Vitar-Sandoval, S. H., Gutiérrez-Maya, J. I., Collazo-Rodríguez, P., y Collazo-Reyes, F. (2018). Determinants of the emergence of modern scientific knowledge in mineralogy (Mexico, 1975-1849): a geohistoriometric approach. Scientometrics ,115(3), 1505-1515. https://doi.org/10.1007/s11192-018-2646-5

Garfield, E. (1972). Citation analysis as a tool in journal evaluation. Science, 178, 471-479.

Garfield, E. (2001). From Computational Linguistics to Algorithmic Historiography. Trabajo presentado en el panel Knowledge and Language: Building Large-Scale Knowledge Bases for Intelligent Applications, Universidad de Pittsburgh, 19 de septiembre. Recuperado de http://garfield.library.upenn.edu/papers/pittsburgh92001.pdf

González-Pereira, B., Guerrero-Bote, V. P., y Moya-Anegón, F. (2010). A new approach to the metric of journals' scientific prestige: The SJR indicator. Journal of Informetrics, 4(3), 379-391. https://doi.org/10.1016/j.joi.201 0.03 .002

Hicks, D., Wouters, P., Waltman, L., de Rijcke, S., y Rafols, I. (2015). El Manifiesto de Leiden sobre indicadores de investigación. Revista Iberoamericana de Ciencia, Tecnología y Sociedad, 10(29), 275-280. Recuperado de http: //www.redalyc.org/pdf/924/92438580012.pdf

Hirsch, J. E. (2005). An index to quantify an individual's scientific research output. Proceedings of the National Academy of Sciences of the United States of America, 102(46), 16569-16572. https://doi.org/10.1073/pnas.050 7655102

LATINDEX-REDALYC-CLACSO-IBICT (2018). Declaración de México a favor del ecosistema Latinoamericano de acceso abierto no comercial. Recuperado de http://www.accesoabiertoalyc.org/declaracion-mexico/

Luchilo, L. J. (2019). Revistas científicas: oligopolio y acceso abierto. Revista Iberoamericana de Ciencia, Tecnología y Sociedad, 14(40). Recuperado de http://ojs.revistacts.net/index.php/CTS/article/view/94/88

Lucio Arias, D., y Leydesdorff, L. (2008). Main\#path analysis and path\#dependent transitions in HistCite ${ }^{\mathrm{rm} \# \text { based }}$ historiograms. Journal of the Association for Information Science and Technology, 59(12),1948-1962. https://do i.org/10.1002/asi.20903

Luhmann, N. (1996). La ciencia de la sociedad. Barcelona: Anthropos.

Martin-Martin, A., Orduna-Malea, E., Thelwall, M., y Delgado López-Cózar, E. (2018). Google Scholar, Web of Science, and Scopus: a systematic comparison of citations in 252 subject categories. Journal of Informetrics, 12(4), 1160-1177. https://doi.org/10.1016/J.JOI.2018.09.002

Merton, R. K. (1973). La sociología de la ciencia: investigaciones teóricas y empiricas. Madrid: Alianza.

Nichols, D. M., y Twidale, M. B. (2017). Metrics for openness. Journal of the Association for Information Science and Technology, 68(4), 1048-1060. https://doi.org/10.1002/asi.23741

Plan S: Accelerating the transition to full and immediate Open Access to scientific publications (2018, septiembre 4). Science Europe. Recuperado de https://www.scienceeurope.org/wp-content/uploads/2018/09/Plan_S.pdf

Piñeres Sus, J. D., Montes, C., y Vélez-Cuartas, G. (2017). Lucha por el reconocimiento en los modelos de medición: el caso de la Universidad de Antioquia. Andamios, 14(34), 259-281. Recuperado de http://www.scielo.org.mx/ scielo.php?script=sci_arttext\&pid=S1870-00632017000200259\&lng=es\&nrm=iso

Poynder, R. (2019). Plan S and the Global South - What do countries in the Global South stand to gain from signing up to Europe's open access strategy? [Mensaje de blog]. LSE Impact Blog. London School of Economics. Recuperado de https://blogs.lse.ac.uk/impactofsocialsciences/2019/03/06/plan-s-and-the-global-south-whatdo-countries-in-the-global-south-stand-to-gain-from-signing-up-to-europes-open-access-strategy/

Suárez-Tamayo, M., Collazo-Reyes, F., y Pérez-Angón, M. Á. (2018). Emerging roles of regional journals in the accreditation of knowledge in tropical medicine: Biomédica and Memorias do Instituto Oswaldo Cruz, 20072015. Scientometrics, 115(3), 1615-1625. https://doi.org/10.1007/s11192-018-2649-2 
Uribe-Tirado, A., y Alhuay-Quispe, J. (2017). Estudio métrico de ALFIN en Iberoamérica: de la bibliometría a las altmetrics. Revista Española de Documentación Cientifica, 40(3), e180. http://dx.doi.org/10.3989/redc.2017.3 .1414

Uribe-Tirado, A., Ochoa-Gutiérrez, J., Pallares Delgado, C., Restrepo Quintero, D., Vélez-Cuartas, G., Robledo Velásquez, J., Gómez Molina, H., Correa Tabares, M. C., Calle Mosquera, J., y Osorio López, A. M. (2019a). Situación del acceso abierto en las universidades. Modelo de análisis y caso Universidad de Antioquia [pre-print].

Uribe-Tirado, A., Ochoa Gutiérrez, J., y Medina Alfonso, D. (2019). Visibilidad de los investigadores de la Universidad de Antioquia en medios de comunicación internacionales, nacionales y regionales-locales [pre-print].

Uribe-Tirado, A., Ochoa Gutiérrez, J., Ruiz-Muñez, K., y Fajardo-Bermúdez, M. (2019). Visibilidad e impacto altmétrico de los investigadores de la Universidad de Antioquia. Metodología aplicable a universidades de América Latina [pre-print].

Uribe-Tirado, A., Vallejo Echavarria, J., y Betancur Marín, D. (2016). Somos Visibles y Tenemos Impacto. Análisis desde Datos de Acceso Abierto, Altmetrics y otros de la Revista Interamericana de Bibliotecología. Revista Interamericana de Bibliotecología, 39(3), 243-275. https://doi.org/10.17533/udea.rib.v39n3a04

Vélez-Cuartas, G. J., Gómez-Flórez, H., Úsuga-Ciro, A., y Vélez-Trujillo, M. (2014). Diversidad y reconocimiento de la producción académica en los sistemas de evaluación de la investigación en Colombia. Revista Española de Documentación Cientifica, 37(3), 1-14. https://doi.org/10.3989/redc.2014.3.1133

Vélez-Cuartas, G. J., Uribe-Tirado, A., Robledo-Velásquez, J., Restrepo, D., Correa, M. C., Pallares, C., González, F., Osorio-López, A. M., Mendoza-González, S., y Castro, A. M. (2017). Indicadores de vinculación con el entorno para Unidades de Gestión de Investigación (Universidad de Antioquia - SIU. Estudio Piloto 2004-2016). Recuperado de http://eprints.rclis.org/32440/

Vélez-Cuartas, G., Lucio-Arias, D., y Leydesdorff, L. (2016). Regional and global science: Publications from Latin America and the Caribbean in the SciELO Citation Index and the Web of Science. El profesional de la información, 25(1), 35-46. https://doi.org/10.3145/epi.2016.ene.05

Wagner, C. (2008). The new invisible college: science for development. Washington: Brookings institution press.

Wilsdon, J., Bar-Ilan, J., Frodeman, R., Lex, E., Peters, I., y Wouters, P. (2017). Next Generation Metrics: Responsible metrics and evaluation for open science. Luxembourg: Publications Office of the European Union. https://doi.o $\mathrm{rg} / 10.2777 / 337729$

Wu, L., Wang, D., y Evans, J. (2019). Large develop and small teams disrupt science and technology. Nature, 566(7744), 378-382. https://doi.org/10.1038/s41586-019-0941-9

\section{Notas}

1 Este documento es parte también de los resultados del proyecto de investigación "Impacto social, científico y actividades de vinculación con el entorno de centros de investigación de la Universidad de Antioquia", financiado por el Comité para el Desarrollo de Investigación de la Universidad de Antioquia. El proyecto y este artículo se enmarca dentro de las actividades del CoLav, como parte de AmeliCA Métricas. Colav es el colaboratorio de ciencias sociales computacionales fundado por la Vicerrectoría de Investigación, el Sistema de Bibliotecas de la Universidad de Antioquia y las Facultades de Ciencias Naturales y Exactas, de Ciencias Sociales y Humanas y la Escuela Interamericana de Bibliotecología. Su propósito es el desarrollo de las ciencias sociales computacionales a través de la docencia, la investigación y la extensión. Una versión preliminar invitada para presentarse en el $3^{\circ}$ Congreso Internacional de Editores Redalyc "Construyendo el modelo de publicación académica del sur global”. Trujillo, Perú, 16-18 mayo 2018. http://congreso.redalyc.org/ocs /public/congresoEditores/index.html

2 Ver "Nuevo récord de autores: 5214 en un artículo de CMS y ATLAS sobre el quark top". Recuperado de https://francis.naukas.com/2019/02/28/nuevo-record-de-autores-5214-en-un-articulo-de-cms-y-atlas-sobre-el-q uark-top/?fbclid=IwAR11FORONz6u-jvZpTlzSBUj1OCgBaTOpl_yy7_lsd8JW_DslfPPhSzKkTQ (Consultado: 28-2-2019)

3 Ver: http://www.amelica.org/index.php/index-entradas/(Consultado: 20-3-2019)

4 Fuente: https://www.scimagojr.com/journalrank.php?country=Latin\%20America. Consultado el 26 de Marzo de 2019 correspondiente al año 2017. 
Palabra Clave (La Plata), abril - septiembre 2019, vol. 8, n² 2, e068. ISSN 1853-9912

5 Ver: Altmetría, influmetría... ;Informetria! http://www.inaecu.com/altmetria-influmetria-informetria/ (Consultado: 15-8-2017) 